\title{
Dermatologie
}

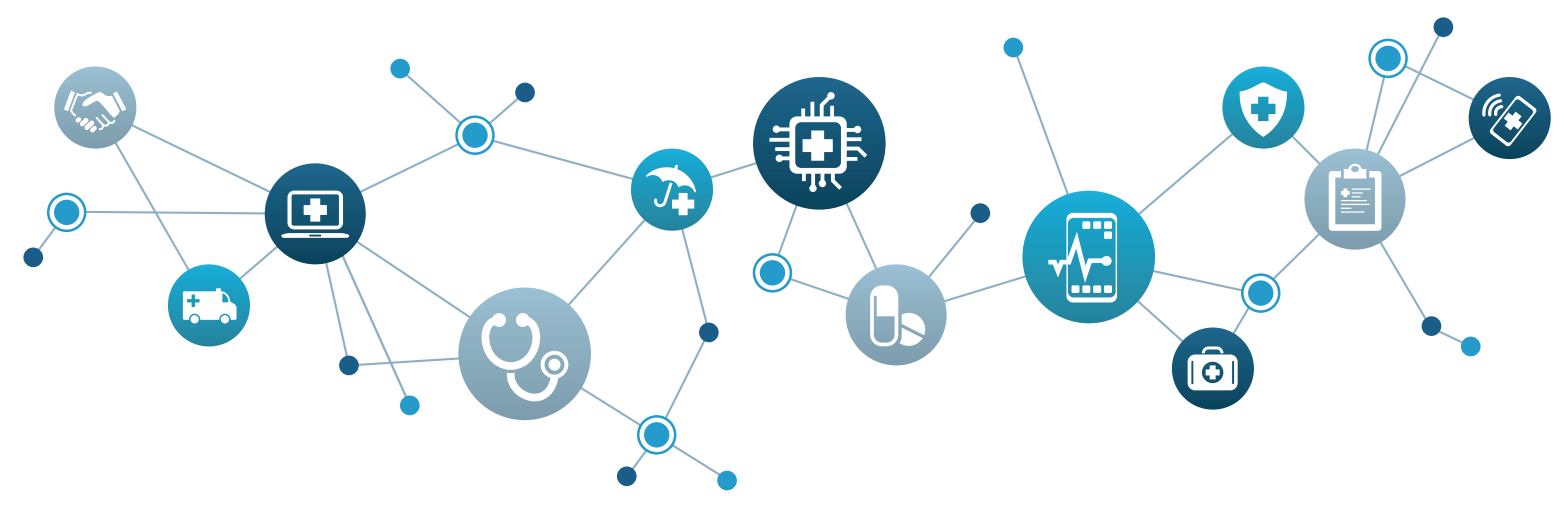

\section{Nahe beim Patienten bleiben}

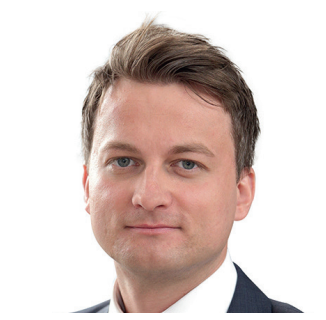

Prof. Dr. David Matusiewicz

FOM Hochschule, KCG KompetenzCentrum für Management im Gesundheits- und Sozialwesen

\section{Was ist}

Viele dermatologische Erkrankungen nehmen einen wiederkehrenden, chronischen Verlauf. Entsprechend muss auch die Behandlung regelmäßig kontrolliert und an den Verlauf bzw. die aktuelle Schwere der Erkrankung angepasst werden. Egal ob Akne, seborrhoisches Ekzem, atopische Dermatitis, Psoriasis oder chronische Wunden: Die Verlaufskontrolle erfolgt dabei aktuell meist im Rahmen eines Präsenztermins, der in mehr oder weniger regelmäßigen Abständen in der Hautarztpraxis erfolgt. Die Verlaufskontrolle im Praxisalltag findet also eher sporadisch und reaktiv statt. Vom Dermatologen kann dann nur der aktuelle Status Quo festgestellt werden, aber evtl. ist der Patient just zum Termin eher Beschwerdefrei und kann auch nur noch lückenhaft und unpräzise etwaige Events zwischen den Arztbesuchen benennen. Selbst wenn die Patienten ein «Tagebuch» führen, sind die dort festgehaltenen Informationen oft lückenhaft, ungenau, schwer nachvollziehbar oder einfach unleserlich. Die Retrospektive auf den Krankheitsverlauf verkommt zum Rätselraten. Eine Digitalisierung der Verlaufskontrolle kann hier ansetzen und für Standards, Regelmäßigkeit und Lückenlosigkeit sorgen. Im Zusammenspiel mit digitalen Scorings wird erstmalig für eine Messbarkeit der Krankheitsverläufe gesorgt und mithilfe von KI lässt sich die Qualität der so erhobenen Daten künftig noch weiter steigern.

\section{Was kommt}

Es wird bald kein Weg mehr vorbei führen an SAPASI, POEM und Co. Die im Oktober 2020 veröffentliche S2k-Leitlinie Telederma- tologie empfiehlt zum Beispiel inzwischen die morphologische Verlaufsdiagnostik der atopischen Dermatitis mittels Teledermatologie sowie die Erhebung Patienten-berichteter Endpunkte wie DLQI und Pruritus. Studien aus den USA belegen, dass die digitale Verlaufskontrolle absolut vergleichbar effektiv ist zu einer Kontrolle beim Präsenztermin in der Praxis. Die Messbarkeit der Krankheitslast jedes einzelnen Patienten wird durch die digitale Erhebung der nötigen Scores ermöglicht. PASI, DLQI, EASI, SCORAD, NAPSI, alle werden ein digitales, vom Patienten selbst erhobenes Pendant bekommen und so steht dann auch einer Verlaufskontrolle von Systemtherapien nichts mehr im Weg. Anwendungen wie die NALA App werden für alle möglichen Erkrankungen und Therapien zur Verfügung stehen und diese für den Patienten dokumentierbar und so für den Arzt jederzeit nachvollziehbar machen. Der Einsatz von KI und Feedback-Funktionen wird die von den Patienten selbst erhobenen Daten genauer, besser auswertbar und lückenloser machen. Alles Gründe, weshalb ebenfalls die Akzeptanz solcher Tools unter den Patienten weiter steigen wird.

\section{Was zählt}

Schließlich kommen wir auch bei der Verlaufskontrolle auf einen ganz wesentlichen Punkt zurück. Es geht nicht darum, dass heute unbedingt jede Behandlung eine digitale Komponente oder Variante braucht. Es geht wie immer darum, dem Patienten die nach aktuellem Wissenstand und Stand der Technik bestmögliche Behandlung zukommen zu lassen. Und dazu bieten die Digitalisierung auch in der Verlaufskontrolle die passenden Ansätze. Diese müssen genutzt und in den Praxisalltag sowie den Alltag der Patienten integriert werden. 


\section{Digitale Verlaufskontrollen in der Dermatologie Eine Einordnung des aktuellen Forschungsstandes und Ausblicke}

Im Praxisalltag findet die Verlaufskontrolle sporadisch, reaktiv und mitunter zufällig statt. Termine in der Sprechstunde erfolgen meist quartalsweise, dabei ist die Entdeckung von Krankheitsveränderungen zeitlich eher zufällig mit dem Praxisbesuch verbunden. Bei der Verlaufskontrolle dermatologischer Erkrankungen erscheint es daher sinnvoll, den Besuch in der Sprechstunde um digitale Angebote zu erweitern. Das macht die systematische Betreuung von Betroffenen möglich. Ein frühzeitiges therapeutisches Eingreifen verbessert den Outcome bei gleichzeitiger Reduktion der persönlichen Kontakte in der Sprechstunde [1, 2].

In der S2k-Leitlinien zur Teledermatologie werden bereits heute telemedizinische Verlaufskontrollen bei Psoriasis, atopischer Dermatitis und chronischen Wunden empfohlen [3].

\section{Forschungsstand zum Einsatz digitaler Verlaufskontrollen}

Der Schwerpunkt der aktuellen Forschung liegt auf den Krankheitsbildern Psoriasis, Akne und atopische Dermatitis [4,5,6]. Randomisierte kontrollierte Studien weisen eine gleichwertige Versorgung durch eine teledermatologische, zeitversetzte Verlaufskontrolle nach. Psoriasis-Erkrankte und ihre Hausärztinnen bzw. ihre Hausärzte konnten in einer US-amerikanischen Studie über eine Teledermatologie-Plattform Anfragen, Bilder und weitere Informationen mit dermatologischem, fachärztlichem Personal teilen. Die Studie zeigt, dass die digitale Verlaufskontrolle gleichermaßen effektiv war [4].

Eine weitere Untersuchung weist nach, dass sich die Lebensqualität von Personen mit Psoriasis bei teledermatologischer Verlaufskontrolle in gleicher Weise verbesserte wie bei Betroffenen, die in der Praxis betreut wurden [7]. Zwei weitere Studien evaluierten den Einsatz der digitalen Verlaufskontrolle bei Personen mit atopischer Dermatitis und Akne, die Informationen direkt an ihre Hautärztin oder ihren Hautarzt sendeten. Bei den Betroffenen mit atopischer Dermatitis zeigte sich ein gleichwertiges klinisches Behandlungsergebnis im Vergleich zur Kontrollgruppe [6]. In einer Untersuchung, die die empfundene Lebensqualität zwischen Betroffenen, die persönlich in die ärztliche Sprechstunde gingen und solchen, die zeitversetzt teledermatologisch betreut wurden, verglich, konnten keine Unterschiede festgestellt werden [8]. Ähnliche Ergebnisse zeigen sich für die digitale Verlaufskontrolle bei Akne [9].

In einem Leitfaden für die deutsche Dermatologie schließen sich die Expertinnen und Experten den Ergebnissen der dargestellten Studien an. Für Neurodermitis, Ekzeme, chronische Wunden und Psoriasis empfehlen sie die Integration der Teledermatologie zur Verlaufskontrolle [10].

\section{Digitale Tools zur Verlaufskontrolle}

Die aktuellen Forschungsergebnisse stützen sich auf sogenannte «Store-and-Forward»-Lösungen (S+F). Dabei handelt es sich um eine Form der zeitversetzten oder asynchronen Telemedizin, bei der Informationen und Bilder an das Behandlungsteam gesendet werden. Die Parteien müssen nicht zur selben Zeit verfügbar sein. Betroffene profitieren von einem ortsunabhängigen und schnellen Zugang zur fachärztlichen Verlaufskontrolle. Der Berufsverband der Deutschen Dermatologen empfiehlt seinen Mitgliedern hierzu die Telemedizin-Plattform von OnlineDoctor, die auch der Autor im klinischen Alltag verwendet. Diese Plattform nutzt aktuell jede zehnte dermatologische Praxis und damit rund 400 Dermatologinnen und Dermatologen. Mit Blick auf die digitale Verlaufskontrolle zeigt sich, dass das persönliche ärztliche Vertrauensverhältnis eine verstärkte Rolle spielt. So präferieren Betroffene in der Regel eine digitale Nachkontrolle bei der Dermatologin oder dem Dermatologen, bei dem sie sich in Behandlung befinden. Genau diese Verknüpfung bietet die Plattform von OnlineDoctor.

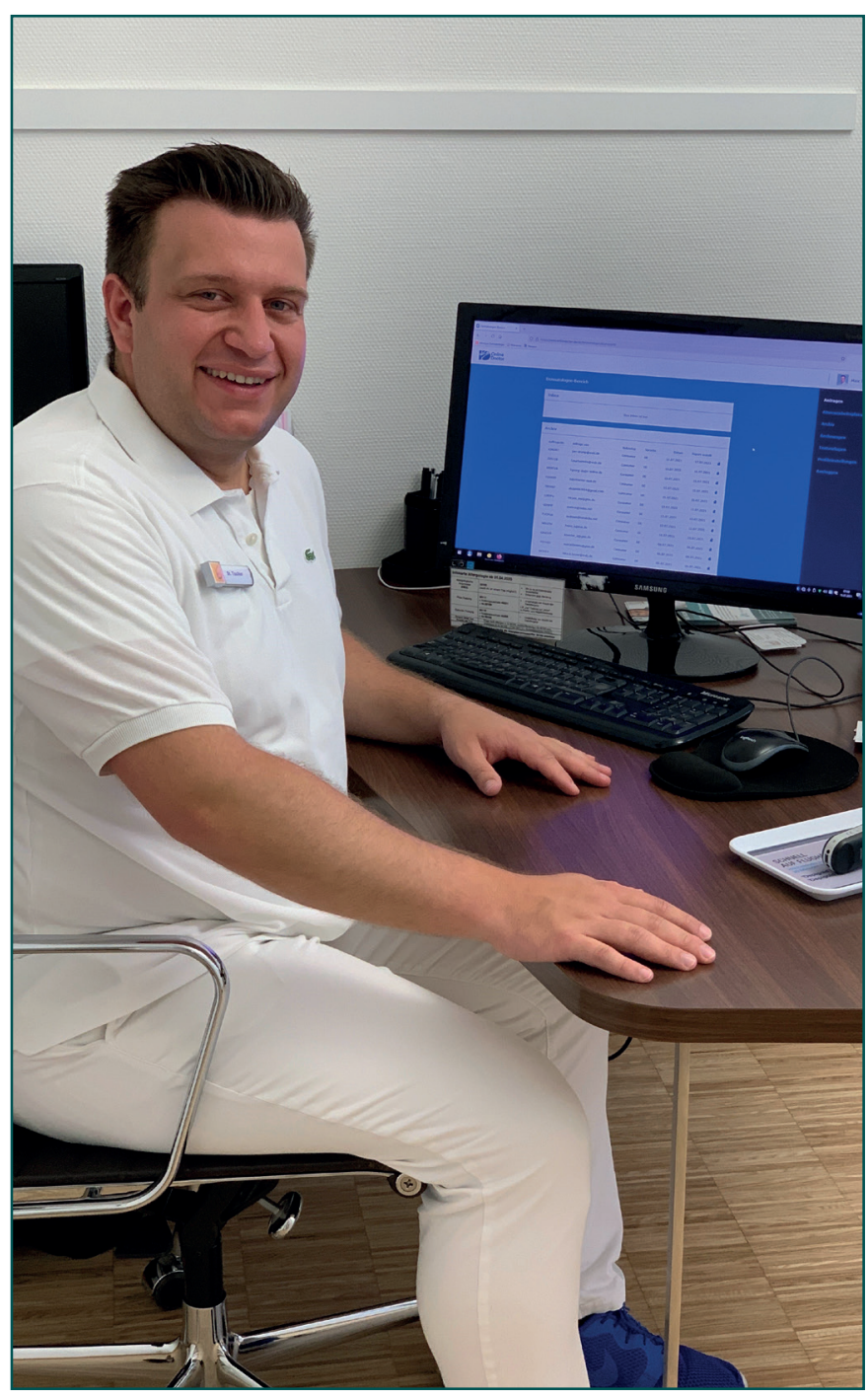

Max Tischler: «Betroffene präferieren in der Regel eine digitale Nachkontrolle bei der Dermatologin oder dem Dermatologen, bei dem sie sich in Behandlung befinden, etwa über die Plattform OnlineDoctor.» 
Aufärztlicher Seite entsteht dadurch eine Veränderung der Nachsorge-Prozesse: Anliegen können zeitnah, flexibel und ortsunabhängig begutachtet werden. Sogenannte hybride Modelle, bei denen digitale Tools die ärztliche Arbeit in der Praxis erweitern, bieten dabei das größte Potenzial. Das Behandlungsteam erkennt schnell unklare Befunde nach einer Initialtherapie. Treten Nebenwirkungen auf, erfolgt digital eine zügige Vorstellung. Ein Beispiel ist die Einleitung einer Systemtherapie bei Akne, wo häufig eine Erstverschlechterung zu Irritation bei Betroffenen führt. Die digitale Kontrolle gibt Sicherheit und erhöht die Therapietreue. Darüber hinaus findet kein Informationsverlust bei der digitalen Verlaufskontrolle nach einer Operation oder während einer Systemtherapie statt, wenn die Akte in der Praxis vorliegt [11]. Im Vergleich zur Videosprechstunde ist die Bildqualität um ein Vielfaches höher.

Die Vorteile der synchronen Telemedizin liegen in der Abrechenbarkeit über den EBM auch bei gesetzlich Versicherten und der direkten Interaktion der Parteien [12]. Die Abrechnung der asynchronen Teledermatologie erfolgt als individuelle Gesundheitsleistung. OnlineDoctor ist derzeit der einzige Anbieter, der mit Krankenkassen kooperiert. Selektivverträge mit aktuell 40 gesetzlichen Kassen ermöglichen ein kostenloses Angebot für 26 Millionen Versicherte in Deutschland.

\section{Aktueller Stand der Messbarkeit (Scores)}

Die Verordnung moderner Systemtherapien erfordert die Messbarkeit der Krankheitsschwere. Hierfür werden analog Messsysteme wie PASI, DLQI, EASI, SCORAD, NAPSI und Co eingesetzt. Ansätze, die Score-Erhebung digital zu gewährleisten, bietet der SelfAdministered Psoriasis Area and Severity Index (SA-PASI) [5]. Bei Betroffenen mit atopischer Dermatitis dient der Patient Oriented Eczema Measurement (POEM) als Messgrundlage [4]. Die Einordnung bei Akne erfolgt digital anhand des Global Acne Severity Scale und der Anzahl der Läsionen (engl. «total lesion count» $[T L C])$. Es liegt damit bereits eine Basis zu digitalen Messbarkeit vor. Nun braucht es Überlegungen, wie auch analoge Scores regelmäßig durch Betroffene selbst oder digital durch ärztliches Personal erstellbar sind und eine Evaluation dieser Scores unter teledermatologischen Bedingungen ermöglicht wird. Beim digitalen Scoring hat zudem der Einsatz Künstlicher Intelligenz (KI) großes Potenzial für die Datenqualität und Umsetzbarkeit in der Praxis.

\section{Evidenzbasiert in die Zukunft}

Die aktuelle Forschung legt nahe, dass die digitale Verlaufskontrolle sowohl bei Betroffenen als auch auf Seite des medizinischen Personals positiv erlebt wird [12]. Für Menschen mit Psoriasis und Atopischer Dermatitis gibt es wissenschaftliche Nachweise dafür, dass sich die Lebensqualität analog zur Verlaufskontrolle vor Ort verbessert. Auch die positiven Effekte wie Einsparungspotenziale bei Warte- und Reisezeit sowie Flexibilität der Kommunikation sind hinreichend belegt. Um im Sinne eines Digital-First-Ansatzes digitale Verlaufskontrollen zum Standard in der Dermatologie werden zu lassen, braucht es weitere Forschung. Die Qualität der digitalen Scorings muss im Vergleich zu analogen Messgrundlagen beurteilt und in prospektiven Studien validiert werden. Nur eine gleichwertige Messbarkeit ermöglicht die Akzeptanz unter Kolleginnen und Kollegen. Das Ziel muss sein, dass die Versorgungsqualität nachweislich verbessert und nicht nur erhalten wird. Teledermatologie-Plattformen bieten hier einen großen Mehrwert: Sie führen anonymisierte Daten aus hunderten dermatologischen Praxen zusammen und können so quantitative Erkenntnisse sammeln - das ist die Grundlage für den Einsatz Künstlicher Intelligenz.

Zeitversetzte Teledermatologie-Plattformen wie OnlineDoctor entwickeln ihre Produkte so weiter, dass Dermatologinnen und Dermatologen effizient und sicher eine Verlaufskontrolle digital anbieten können. Zentral hierfür ist, dass eine Software nicht nur die Begutachtung eines akuten Falls ohne Akte in der Praxis erlaubt, sondern auch wiederkehrende Fälle optimal mit dem Programm visualisiert und bearbeitet werden können.

\section{Literatur}

1. Tunder R., Plein J. (2016) Patient Empowerment als wirksames Instrument zur Steigerung der Behandlungsqualität. In: Pfannstiel M., Rasche C., Mehlich H. (eds) Dienstleistungsmanagement im Krankenhaus. Springer Gabler, Wiesbaden. https://doi. org/10.1007/978-3-658-08429-5_14

2. Samoocha D, Bruinvels DJ, Elbers NA, Anema JR, van der Beek AJ: Effectiveness of Webbased Interventions on Patient Empowerment: A Systematic Review and Meta-analysis. J Med Internet Res 2010;12(2):e23. doi: 10.2196/jmir.1286

3. Deutsche Dermatologische Gesellschaft e.V. (DDG). S2k-Leitlinie Teledermatologie. Im Internet: www.awmf.org/leitlinien/detail/ll/013-097.html.; Stand: 26.11.2021

4. Armstrong AW, Johnson MA, Lin S, et al. Patient-centered, direct-access online care for management of atopic dermatitis: a randomized clinical trial. JAMA Dermatol. 2015;151(2):154-160. doi: 10.1001/jamadermatol.2014.2299.

5. April W. Armstrong1, Adam R. Ford1, Cindy J. Chambers2, Emanual Maverakis2, Cory A. Dunnick3, Mary-Margaret Chren4, Joel M. Gelfand5, Caitlin M. Gibbons1, Brittany M. Gibbonsl and Christianne J. Lanel.: Online Care Versus In-Person Care for Improving Quality of Life in Psoriasis: A Randomized Controlled Equivalency Trial November 24, 2018 DOI: https://doi.org/10.1016/j.jid.2018.09.039

6. Frühauf J, Kröck S, Quehenberger F, et al. Mobile teledermatology helping patients control high-need acne: a randomized controlled trial. J Eur Acad Dermatol Venereol. 2015;29(5):919-924. doi: 10.1111/jdv.12723.

7. Armstrong AW, Chambers CJ, Maverakis E, et al. Effectiveness of Online vs in-person care for adults with psoriasis: a randomized clinical trial. JAMA Netw Open. 2018;1(6):e183062. doi: 10.1001/jamanetworkopen.2018.3062.

8. Heather Kornmehl 1, Sanminder Singh 2, Mary Ann Johnson 3, April W Armstrong 45 Direct-Access Online Care for the Management of Atopic Dermatitis: A Randomized Clinical Trial Examining Patient Quality of Life. Telemed J E Health. 2017 Sep;23(9):726732. doi: 10.1089/tmj.2016.0249. Epub 2017 Jun 1.

9. Alice J Watson 1, Hagit Bergman, Christy M Williams, Joseph C Kvedar: A randomized trial to evaluate the efficacy of online follow-up visits in the management of acne. Arch Dermatol 2010 Apr;146(4):406-11.doi: 10.1001/archdermatol.2010.29.

10. Augustin M et al: Praxis der Teledermatologie. Leitfaden der deutschsprachigen Dermatologen. JDDG. 2018. DOI: 10.1111/ddg.13512

11. Reinders $\mathrm{P}$, Otten M, Augustin $\mathrm{M}$ et. al. Anwendungsbereiche der Teledermatologie. Hautarzt 2021. doi:10.1007/s00105-021-04917-y e.

12. Kassenärztliche Bundesvereinigung (KBV), Coronavirus: Videosprechstunden unbegrenzt möglich.ImInternet:https://www.kbv.de/html/1150_44943.php;Stand:26.11.2021

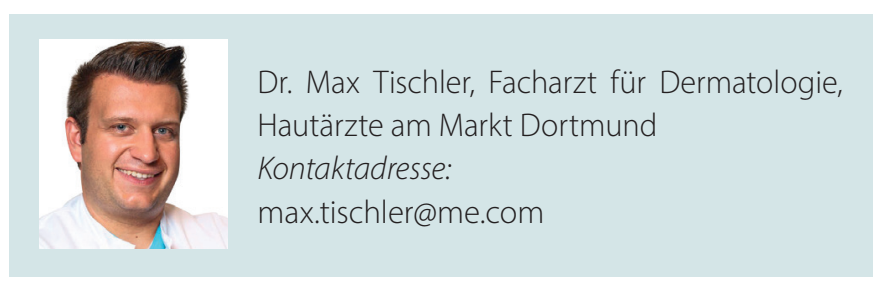

34

Kompass Dermatol 2022;10:32-39 DOI: $10.1159 / 000522270$ 


\section{Digitale Verlaufskontrolle Wie Apps die Behandlung der atopischen Dermatitis unterstützen}

Dieser Artikel beleuchtet die Verlaufskontrolle bei Patienten mit atopischer Dermatitis näher. Dabei werden zunächst der Status Quo beim «Standard of Care» (SOC) sowie bestehende Herausforderungen im Praxis- und Patienten-Alltag vorgestellt. Anschließend soll erklärt werden, wie digitale Lösungen dabei helfen können, diese Herausforderungen zu meistern.

\section{Status Quo}

Die atopische Dermatitis ist eine entzündliche Erkrankung der Haut, häufig mit chronisch-rezidivierendem Verlauf. Die Erstmanifestation ist häufig bereits im frühen Kindesalter und stellt die häufigste chronische Erkrankung in diesem Lebensabschnitt dar. Jedoch kann sie sich auch erst im (höheren) Erwachsenenalter manifestieren. Die Ursachen der atopischen Dermatitis sind multifaktoriell. Die genetische Veranlagung wie auch Allergien als mögliche Triggerfaktoren spielen eine Rolle.

Betroffene besuchen abhängig vom Schweregrad der Erkrankung ihr «Care-Team», welches aus Experten sehr vieler verschiedener Fachrichtungen bestehen kann: Dermatologen, Allergologen, Ernährungsexperten, Psychologen, Psychodermatologen, gesamtheitliche/integrative Mediziner, Kinderärzte, Hausärzte etc.

Es ist besonders wichtig, dass bei der Erstvorstellung eine ausführliche Anamnese erfolgt. Abhängig vom Arzt folgt dann in den anschließenden Terminen eine ausführliche Verlaufskontrolle, bei der der Hautstatus, verwendete Cremes bzw. der aktuelle Therapieplan und evtl. sogar dermatologischeScoreswie der(Children's)

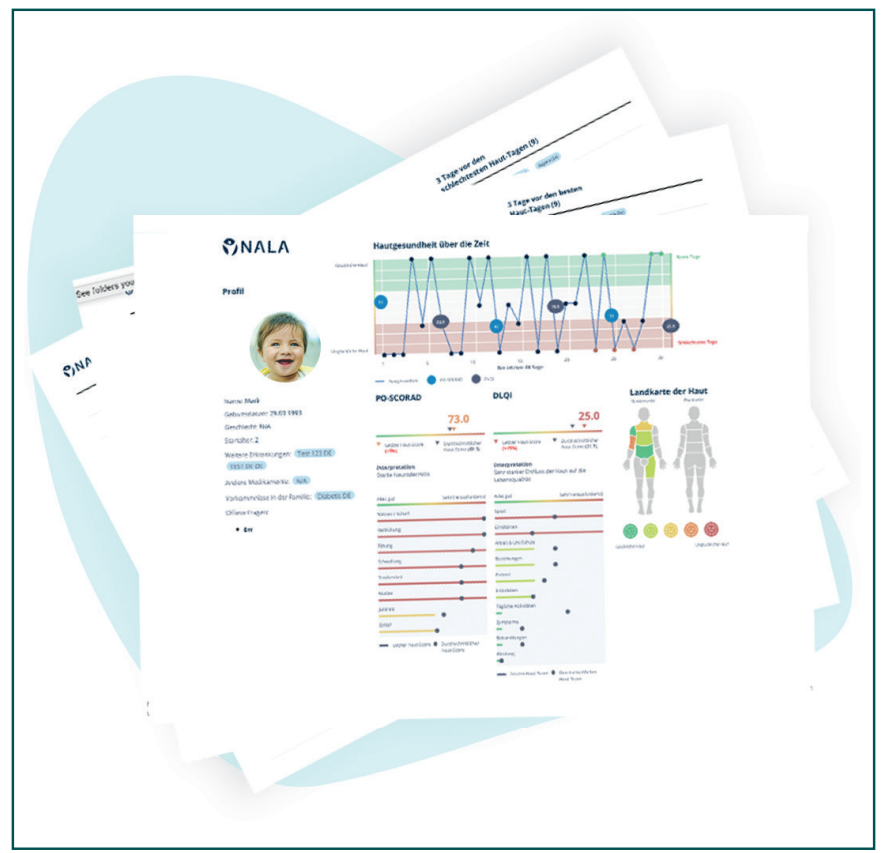

Über einen visuell sehr ansprechenden, 8-seitigen Haut-Report (PDF) kann der behandelnde Arzt den Hautstatus des Patienten auf einen Blick erfassen.

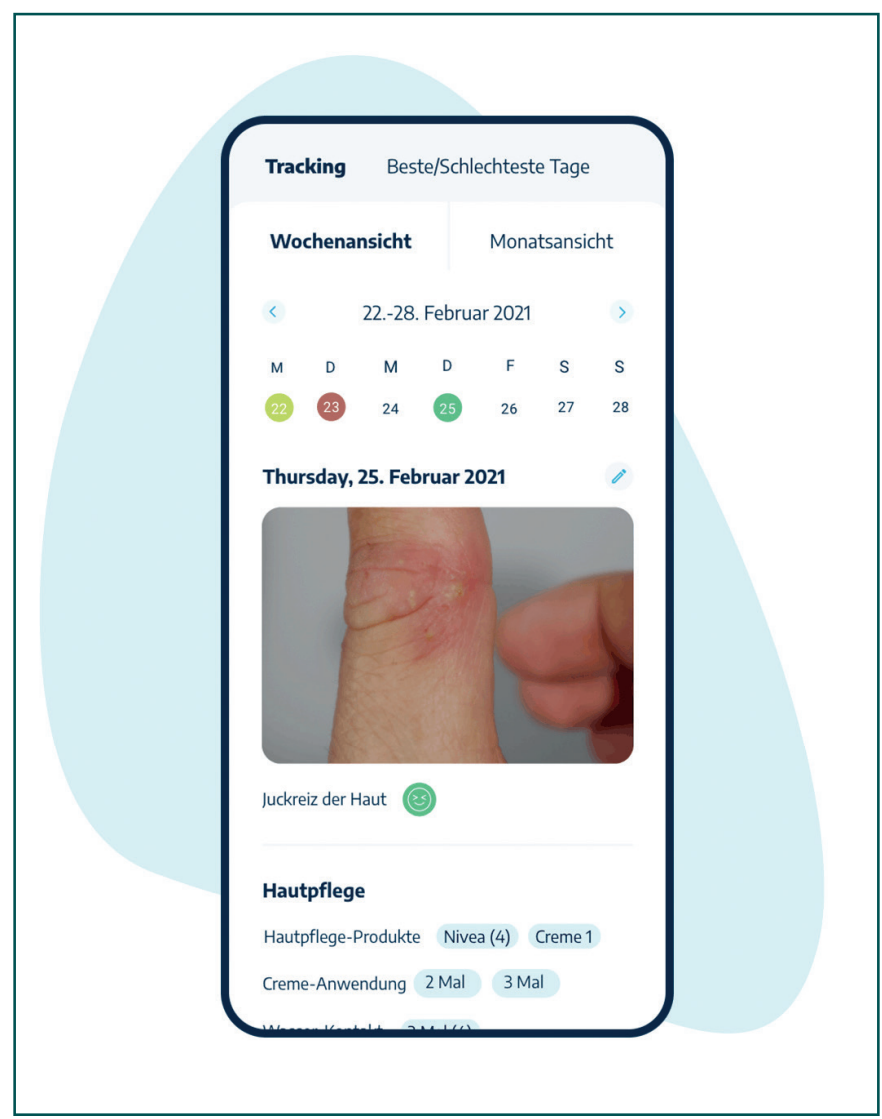

Durch das tägliche Tracking der Hautgesundheit und möglicher Einflussfaktoren, können fast spielerisch neue Schubauslöser (DONTs) und Hautunterstützer (DOs) entdeckt werden.
Dermatology Life Quality Index ((C)DLQI) oder der Patient Oriented SCORAD ((PO-)SCORAD) erfasst werden. Die S2k-Leitlinie Teledermatologie empfiehlt inzwischen die morphologische Verlaufsdiagnostik der atopischen Dermatitis mittels Teledermatologie sowie die Erhebung Patienten-berichteter Endpunkte wie DLQI und Pruritus. Die Leitlinienautoren sind sich einige, dass die atopische Dermatitis, bei erfolgter Diagnosestellung durch eine Präsenzuntersuchung, grundsätzlich mit einer Store-and-Foreward oder Realtime-Technologie im Verlauf begutachtet werden kann.

\section{Digitale Unterstützung im Praxisalltag}

Die Verlaufskontrolle ist für den Arzt häufig mit sehr vielen Fragen verbunden, die er zu den vergangenen Wochen/Monaten seit dem letzten Arzttermin stellt, um ein genaues Bild über den Zustand der Haut zu erhalten. Dem Patienten empfohlen werden kann dazu das Führen eines Ernährungs-, Behandlungs- und Allergen-Tagebuches (oder Ähnlichem), je nachdem, in welchem Bereich Schubauslöser vermutet werden bzw. welche Bereiche besondere Aufmerksamkeit benötigen. Im Idealfall würde der Arzt vor jedem Arztbesuch einen Report über den Zustand der Haut 
des Patienten bekommen, in dem er übersichtlich und im Zeitverlauf sehen könnte. .

- ...wie sich gewisse Scores über die Zeit entwickeln (bspw. Juckreiz, (PO-)SCORAD, (C)DLQI oder EASI).

- ...welche Hautstellen besonders betroffen sind, bspw. auf einer Art «Haut-Landkarte».

- ...wie die Haut zwischen den Arztbesuchen aussieht, bspw. über eine Foto-Dokumentation.

- ...welche Fragen den Patienten beschäftigen.

\section{Digitale Unterstützung im Patienten-Alltag}

Aktuell ist das Dokumentieren der Haut via Foto sowie bestimmter Schubauslöser (wie bspw. Ernährung, Allergene) oder bestimmter sonstiger Einflussfaktoren (wie bspw. Cremes und Medikamente) sehr mühsam. Fotos werden händisch mit dem Smartphone oder der Fotokamera aufgenommen und schwirren ungeordnet und unstrukturiert im Foto-Album umher. Schubauslöser und andere Einflussfaktoren müssen ganz «old school» via Stift und Papier aufgenommen werden und gehen entweder verloren oder sind so unleserlich, dass sie vom Arzt nicht entziffert werden können. Auch sind keine Analysen (bspw. via einfachen statistischen Regressionen) möglich, um etwa neue Schubauslöser zu identifizieren.

\section{Vorteile der digitalen Verlaufskontrolle}

Die beiden Gründer Jeremy und Spencer wollen das mit NALA ändern. Sie haben NALA aus eigener Betroffenheit gegründet: Je-

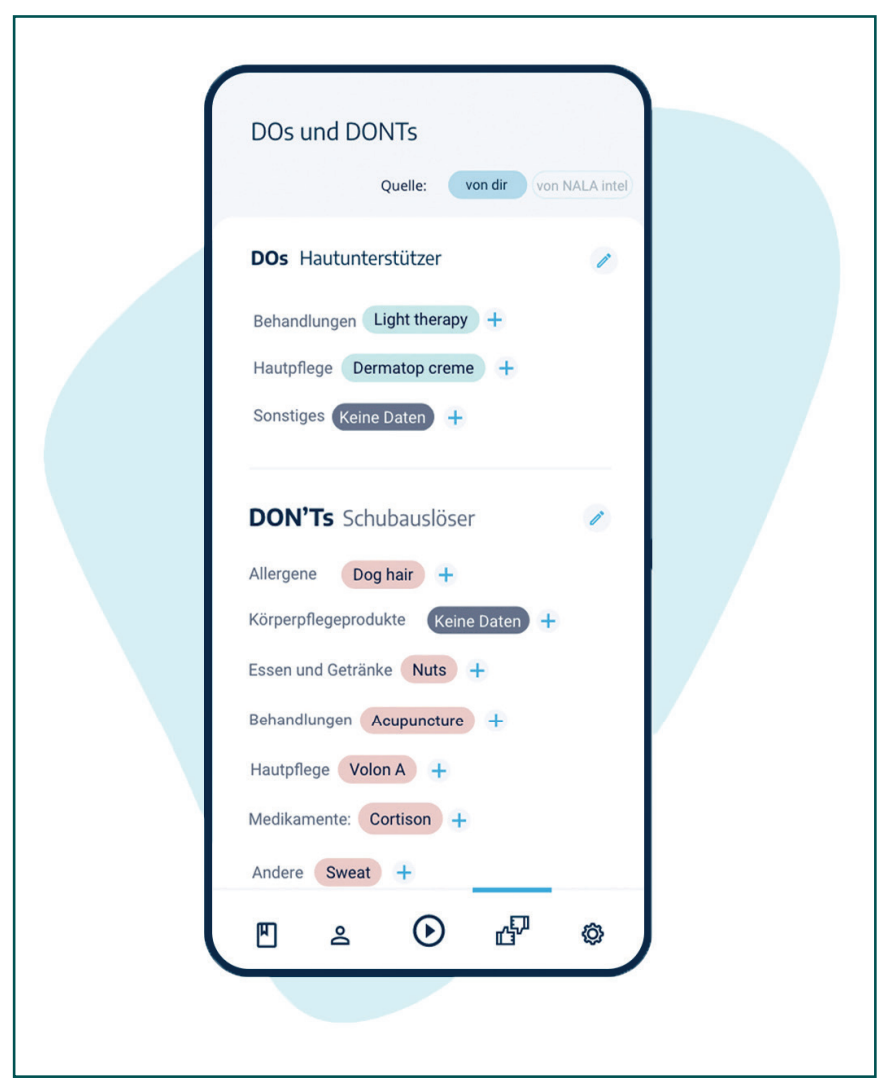

Basierend auf dem täglichen Haut-Tracking und statistischen/KI-Modellen, kann der Therapieplan (d.h. DOs und DONTs) viel schneller personalisiert werden. remy litt als Kind und Jugendlicher selbst so stark unter Neurodermitis, dass er einbandagiert wurde und über Nacht Stoffhandschuhe anziehen musste, damit er sich nicht kratzte. Spencer's Sohn Joshua (heute 4 Jahre alt) leidet aktuell an Neurodermitis. NALA ist also von Patienten für Patienten entwickelt - unterstützt durch Key Opinion Leader wie beispielsweise Prof. Dr. Marcus Maurer von der Charité in Berlin.

Die Verlaufskontrolle der atopischen Dermatitis per NALA App funktioniert so: Betroffene (bzw. deren Eltern) können via der NALA App ganz einfach Fotos von bestimmten Körperstellen machen und diese - gemeinsam mit einer Angabe des Schweregrades - am entsprechenden Körperbereich ablegen. So entsteht eine übersichtliche «Landkarte der Haut», auf der man über ein Ampel-System (grün - gelb - rot) direkt visuell erfassen kann, welche Hautstellen am meisten betroffen sind. Außerdem kann man in die einzelnen Hautstellen «hineinklicken» und so im zeitlichen Verlauf die abgelegten Fotos der Hautstelle verfolgen.

Darüber hinaus können pro Tag alle denkbaren Hautunterstützer und Schubauslöser (d.h. Cremes, Medikamente, Cremehäufigkeit, Ernährung, Allergene, Schlaf, Stress etc.) sowie Scores für die Hautgesundheit selbst aufgezeichnet werden. Auch offene Fragen können erfasst werden, sowie die persönlichen DOs (d.h. Hautunterstützer) und DONTs (d.h. Schubauslöser), sodass man immer übersichtlich parat hat, welche Dinge man häufiger tun sollte (DOs) und welche Schubauslöser man eher meiden sollte (DONTs).

Diese Daten können dann über einen Klick in einen 8-seitigen PDF-Report übertragen werden, in dem graphisch übersichtlich und visuell ansprechend alle wichtigen Informationen verfügbar sind. Dieser Report kann dem Arzt zugesandt werden, sodass alle wichtigen Informationen einfach und intuitiv erfasst werden können.

Der Vorteil für den Arzt ist, dass in der Zeit, in der sich der Patient entkleidet, damit der Arzt einen Blick auf die Haut werfen kann, er bereits alle wichtigen Informationen und Fragen erfassen kann, und dann direkt mit der Behandlung loslegen kann - und eben nicht erst über viele Fragen sich ein unvollständiges Bild des Hautzustandes des Patienten erarbeiten muss.

Als positiver Nebeneffekt ist sich auch der Patient bewusster, wie häufig er sich eincremt, Stichwort: Medikations-Adhärenz, und welche Nahrungsmittel er zu sich nimmt, welchen Allergenen er ausgesetzt ist und wie gut sein Stresslevel und seine Schlafqualität sich über die Zeit entwickeln. Alleine dadurch stellen sich bereits Effekte im Verhalten des Betroffenen ein, sodass gewisse Anpassungen im Lifestyle des Patienten für eine glücklichere Haut auch ganz ohne Arztintervention geschehen.

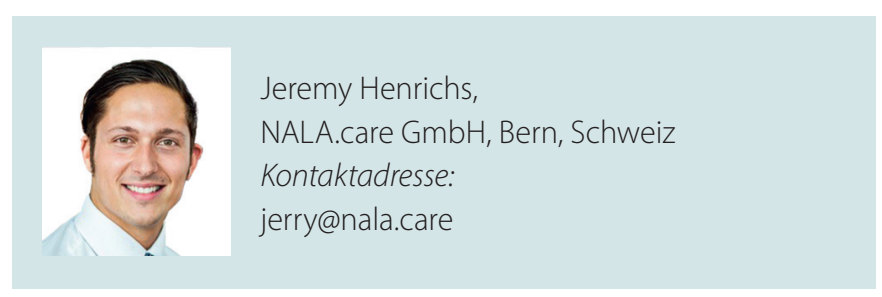




\section{Apollon-Hochschule Neue Professur «Digital Health» geht an Felix Hoffmann}

Prof. Dr. Felix Hoffmann ist seit Anfang des Jahres an der Apollon Hochschule Kursleiter für «Digital Health», ein Thema, das aus dem Gesundheitswesen nicht mehr wegzudenken ist. In seinem Hauptberuf ist Felix Hoffmann Arzt mit Facharztausbildung für Orthopädie und Unfallchirurgie. «Als Health Manager und Experte in Medizinrecht und -didaktik sowie seinem Know-how in Medizin 4.0 und digitaler Transformation, ist der neue Kollege bestens prädestiniert, den Ruf auszufüllen», freut sich Präsidentin Prof. Dr. Johanne Pundt. Dabei versteht Professor Hoffmann unter digitaler Transformation und Medizin 4.0 einen in digitalen Technologien begründeten gesellschaftlichen Veränderungsprozess, der mit einem Wandel der Werteordnung einhergeht und weit über die Entwicklung und Nutzung digitaler Anwendungen hinausgeht. In der Vergangenheit beschäftigte er sich unter anderem mit Projekten zur Weiterentwicklung klinischer Prozesse unter dem Einsatz digitaler Technologien, dem Aufbau einer Infrastruktur für die Zentralambulanz, die Telematikinfrastruktur und die Reorganisation der Notaufnahme einschließlich der Implementie- rung der ZNA-Software Epias. Neben seiner aktuellen Professur leitet Felix Hoffmann die Stabstelle für medizinische Prozessentwicklung am Klinikum Darmstadt und verantwortet dort in enger Zusammenarbeit mit allen Abteilungen des Klinikums und insbesondere der IT-Abteilung die Neuaufstellung der analogen und digitalen Prozesslandschaft.

SeitMitte JanuarhataußerdemDr.Claudia Kemper die Professur «Versorgungsforschung - Schwerpunkt Therapie und Pflege» inne an der Apollon Hochschule. Die ausgebildete Physiotherapeutin, Religionspädagogin und promovierte Gesundheitswissenschaftlerin arbeitete bereits seit März 2021 als wissenschaftliche Mitarbeiterin an der Hochschule und widmet sich nun der Weiterbildung der Therapieberufe. «Die neue Kollegin», freut sich die Präsidentin Prof. Dr. Johanne Pundt, «vervollkommnet mit ihren fundierten Qualifikationen und wissenschaftlichen, medizin-therapeutischen und pädagogischen Erfahrungen die Vielfalt der Gesundheitsberufe, die die APOLLON Hochschule einerseits intern vertritt und andererseits auch nach außen anbietet.»

Professorin Kemper war bereits als Lehrkraft an verschiedenen Hochschulen, als Schul-

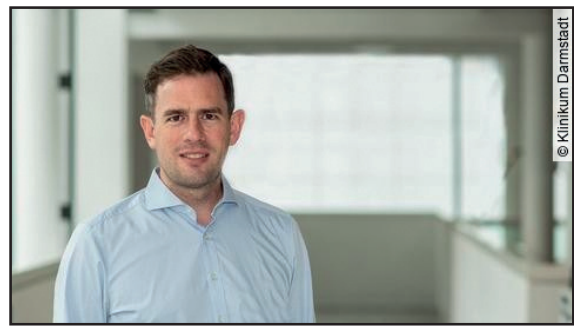

Dr. Felix Hoffmann ist neben seiner Tätigkeit am Klinikum Darmstadt auch Professor für Digital Health an der ApolIon Hochschule in Bremen. Foto: Klinikum Darmstadt

leitung und als Geschäftsführung eines Hospiz- und Palliativzentrums tätig und hat ebenso in der Berufspolitik als Generalsekretärin des Deutschen Verbandes für Physiotherapie, an der internationalen Vernetzung, Leitlinienarbeit und an Ausbildungsfragen aktiv mitgewirkt. Für ihren wissenschaftlichen Schwerpunkt der Versorgungsforschung bringt sie Erfahrungen in Innovationsfondprojekten mit. Claudia Kemper möchte sich auf Impulse aus den Gesundheitsfachberufen heraus konzentrieren, um die Weiterentwicklung akademischer Strukturen sowie die Vernetzung von Wissenschaft und Praxis mitzugestalten.

\section{Digitalisierung im Gesundheitswesen Skepsis von Ärztinnen und Ärzten überwinden}

Die Digitalisierung im Gesundheitswesen stößt weiterhin bei Ärztinnen und Ärzten auf große Skepsis. Das zeigt der Anfang 2022 veröffentlichte Digitalisierungsreport 2021 von DAK-Gesundheit und Ärzte Zeitung. Etwa zwei Drittel der Befragten fühlt sich nicht ausreichend auf die Nutzung digitaler Gesundheitslösungen wie der elektronischen Patientenakte vorbereitet. «Die Ergebnisse zeigen, dass die Ärzteschaft bei der Digitalisierung nicht mitgenommen wurde,» sagt Andreas Storm, Vorstandsvorsitzender der DAK-Gesundheit. «Wir brauchen deshalb eine gemeinsame Digitalisierungsstrategie und eine neue Qualität der Zusammenarbeit.» Nur gemeinsam könne die Digitalisierung erfolgreich sein. Der Report zeigt auch: Wer digitale Anwendungen bereits nutzt, sieht vielfach deren Vorteile. 569 Ärztinnen und Ärzte sowie 16 Psychotherapeutinnen und -therapeuten haben sich im Frühherbst 2021 an der Online-Befragung von E-Patient Analytics beteiligt und sich zu ihren Erfahrungen und Einstellungen im Bereich E-Health geäußert. Der Großteil der Befragten kennt die wichtigsten digitalen Gesundheitslösungen, hatte sie zum Zeitpunkt der Befragung aber noch nicht eingesetzt. Fast die Hälfte der Befragten fühlt sich mit der Nutzung der digitalen Anwendungen überfordert. «Ärztinnen und Ärzte arbeiten wegen der Pandemie bereits am Anschlag», sagt Hauke Gerlof, stellvertretender Chefredakteur der Ärzte Zeitung. «Da ist es kein Wunder, dass die Digitalisierung mit neuen Anwendungen wie eAU-Bescheinigung, E-Rezept und elektronische Patientenakte Schwierigkeiten bereitet. Dabei ist die Technik bei der Einführung häufig noch fehlerhaft, Updates führen zum Absturz der Systeme, die Anzahl der Fehlermeldungen war zu Beginn abschreckend.» Insofern sei die zunächst ablehnende Haltung vieler Ärztinnen und Ärzte nicht überraschend.

Die am weitesten verbreitete digitale Anwendung in Arztpraxen ist laut Digitalisierungsreport 2021 die elektronische Ter- 
minvergabe, die von knapp 15 Prozent der befragten Medizinerinnen und Medizinern regelmäßig genutzt wird und von weiteren 14 Prozent bereits verwendet worden ist. Von den Befragten mit Nutzungserfahrung bescheinigen ihr 64 Prozent eine Zeitersparnis in der Praxisorganisation. Dies deckt sich mit dem Befund der Studie, dass gut die Hälfte der befragten Ärztinnen und Ärzten, die bereits Erfahrungen mit digitalen Gesundheitslösungen gesammelt haben, auch deren Vortei- le sehen: 56 Prozent erkennen beispielsweise im Einsatz eines elektronischen Medikationsplans eine verbesserte Qualität der Patientenversorgung.

Besondere Skepsis ermittelte die Studie in Bezug auf die Telematikinfrastruktur (TI). Diejenigen Befragten, die ihre Eindrücke in einem Freitextfeld schilderten, äußerten sich zu 93,5 Prozent negativ zu ihren Erfahrungen mit der $\mathrm{TI}$. Viele bemängelten eine ungenügende Einbindung der Ärzteschaft seitens Politik und Gematik und erklärten, sich bevormundet zu fühlen. «Die Ergebnisse unseres Digitalisierungsreports sind ein Weckruf, der Konsequenzen haben muss,» betont DAK-Vorstand Storm. Seine Forderung lautet: «Wir brauchen eine neue Qualität der Zusammenarbeit. Entscheidend wird sein, sowohl die Ärztinnen und Ärzte als auch die Krankenkassen stärker einzubinden und die Digitalisierung mit ihnen gemeinsam zu gestalten - orientiert am Versorgungsalltag und am Nutzen für die Patientinnen und Patienten», sagt der Kassenchef.

\section{Stiftung Gesundheit Apps auf Rezept etablieren sich}

Ein Jahr nach der Einführung sind verschreibungsfähige Digitale Gesundheitsanwendungen (DiGA) auf dem Weg, ein etablierter Teil der Gesundheitsversorgung zu werden. Zu diesem Ergebnis kommt die repräsentative Studie «Ärztinnen und Ärzte im Zukunftsmarkt Gesundheit 2021/2», die die Stiftung Gesundheit Mitte Dezember veröffentlicht hat. „Mehr als 80 Prozent der Ärzte sind mittlerweile mit den Apps auf Rezept vertraut", berichtet Prof. Dr. Dr. Konrad Obermann, Forschungsleiter der Stiftung Gesundheit. „Und etwa jeder fünfte Arzt hat bereits praktische Erfahrungen mit diesem neuen Instrument gesammelt."

\section{Wirksam bei gezieltem Einsatz}

Den Apps auf Rezept stehen Ärzt:innen grundsätzlich positiv gegenüber: Zwei Drittel von ihnen sind der Meinung, dass gezielt eingesetzte Apps überaus hilfreich sein können. Den größten Nutzen sehen Ärzt:innen derzeit bei Tagebuchanwendungen (82,7 Prozent), gefolgt von Apps zur Ernährungsberatung (77,3 Prozent), zur Aufzeichnung von Vitalparametern sowie zur Verhaltenskontrolle (jeweils 76,7 Prozent). Kritisch sehen sie dagegen einem Einsatz von DiGA in psychischen Anwendungsbereichen wie Depressionen oder Suchtverhalten: In diesen Bereichen halten weniger als 40 Prozent einen Einsatz für sinnvoll.

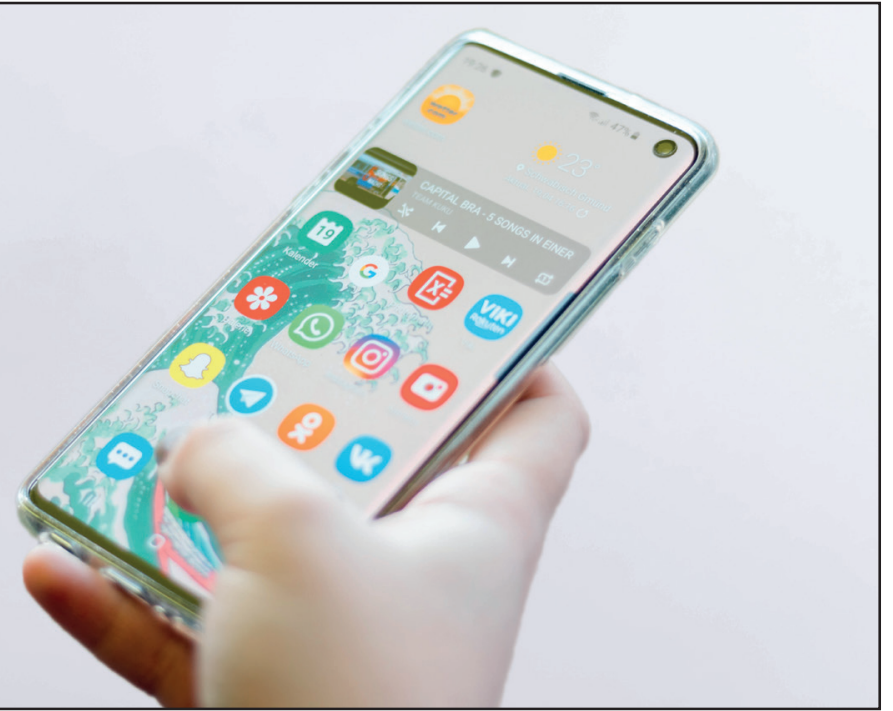

Apps werden auch in der Gesundheitsversorgung immer beliebter und bekannter. Foto: Christian Wiediger/Unsplash

\section{Studie identifiziert Hürden}

In der aktuellen Studie quantifiziert die Stiftung Gesundheit erstmals auch die Hürden, die den Einsatz von DiGA aus Sicht der Ärzte derzeit erschweren: „Mit rund 70 Prozent stehen hier datenschutzrechtliche Bedenken klar an der Spitze", berichtet Obermann. Zudem hätten jeweils knapp die Hälfte der Responder Zweifel an der Wirksamkeit, Zweifel an der Patienten-Motivation oder bemängelten fehlende Testmöglichkeiten für Behandler:innen. Organisatorische Hürden dagegen stellten nur noch für knapp ein Drittel der Ärzt:innen ein Hemmnis dar: „Das spricht dafür, dass in diesem Bereich alle Beteiligten ihre Hausaufgaben gemacht haben", so Obermann.

Als größte Hemmnisse für den Einsatz von DiGA sehen Ärzt:innen neben Datenschutzbedenken Zweifel an der Wirkung sowie an der Motivation der Patient:innen, Apps auf Rezept zu nutzen.

Die aktuelle Studie ist Teil der Studienreihe "Ärztinnen und Ärzte im Zukunftsmarkt Gesundheit", mit der die Stiftung Gesundheit seit 2005 Trends und Entwicklungen im Gesundheitssektor untersucht. Für die aktuelle Ausgabe befragte die Stiftung Gesundheit ein repräsentatives Sample ambulant tätiger Ärzt:innen und Psychologischer Psychotherapeut:innen und verglich die Ergebnisse mit der Vorgängerstudie aus dem Jahr 2020. 


\section{Beiersdorf \\ Beiersdorf investiert in Start-up für digitale Hautarztbesuche}

Der Hautpflegekonzern Beiersdorf investiert über seine OSCAR\&PAUL Venture Capital Unit in das Düsseldorfer Start-up dermanostic, wie Anfang Januar 2022 gemeldet wurde. Das junge Unternehmen bietet im strategisch wichtigen Feld «Digital Health» dermatologische Diagnosen per App an. Der Service ist ortsunabhängig und rund um die Uhr für 25 Euro zu buchen - die Diagnosen erfolgen durch erfahrene Dermatolog*innen binnen 24 Stunden, nachdem Patient*innen drei Fotos hochgeladen und einen AnamneseFragebogen ausgefüllt haben. Gerade bei leichteren Fällen kann dermanostic so den Besuch in einer dermatologischen Praxis komplett ersetzen und schnelle Lösungen für individuelle Hautprobleme liefern, beispielsweise durch den Versand eines benötigten Rezepts per Post oder digital über Online-Apotheken.

Ascan Voswinckel, Head of OSCAR\&PAUL Beiersdorf Venture Capital, sieht in dem jüngsten Investment und der damit einhergehenden Kollaboration mit dermanostic einen wichtigen Schritt. «Wir glauben an das große Potenzial von ,Digital Health' und Teledermatologie - gerade in der jüngeren Zielgruppe. Digitale Besuche bei Dermatolog*innen bieten unseren Verbraucher*innen einen großen Mehrwert, denn sie ermöglichen eine schnelle, unkomplizierte, kostengünstige und professionelle Beratung für ihre Hautindikation. Damit passt das neue digitale Angebot von dermanostic perfekt zu unserer Marke Eucerin - und es ergänzt die digitale Consumer Journey unserer Dermokosmetik-Marke auf innovative Weise.» Das bestätigt auch Cornelius Becker, Leiter des Bereichs Derma bei Beiersdorf: «Die Zusammenarbeit mit dermanostic ist ein relevanter Aspekt unserer Digitalisierungsstrategie. Besonders in Zeiten von COVID-19 müssen wir digitale Wege finden, um unseren Konsument*innen trotz physischer Distanz die passende Beratung zur Lösung ihrer Hautprobleme zu bieten. Durch den engen Austausch mit den Expert*innen von dermanostic können wir voneinander lernen und somit noch besser digitale Nähe zu unseren Verbraucher*innen schaffen.» Becker erklärt weiter: «Hautprobleme haben nicht immer nur Auswirkungen auf unsere Haut oft beeinflussen sie auch das Selbstbewusstsein, die Gefühlslage und damit die Lebensqualität der betroffenen Personen. Doch mit den richtigen dermatologischen Lösungen kann geholfen werden. Hierbei setzen wir neben unserer intensiven Forschungs- und Entwicklungsarbeit auch auf den engen Austausch mit Dermatolog*innen.»

«Wir freuen uns, mit Beiersdorf einen starken und erfahrenen Partner an unserer Sei- te zu haben», so Dr. Ole Martin, Geschäftsführer und Mitgründer von dermanostic, zum Investment von Beiersdorf. «Schon in den zurückliegenden Monaten konnten wir feststellen, wie gut dermanostic und Eucerin zusammenpassen. Im Rahmen der Kooperation finden interessierte Nutzer*innen Informationen über unsere Angebote und Leistungen auf einer dezidierten Landingpage über www.eucerin. de. Nutzer*innen mit Hautproblemen haben hier auch direkt die Möglichkeit zum Service von dermanostic zu gelangen, um eine Hautberatung zu erhalten. Ein Angebot, das gut angenommen wurde.»

Im Sinne der Gründungsväter von Beiersdorf, Oscar Troplowitz und Paul Beiersdorf, investiert die OSCAR\&PAUL Beiersdorf Venture Capital Unit in innovative Unternehmen und Start-ups. Die Einheit verfolgt dabei das Ziel, gegenseitigen Wert zu schaffen und gemeinsam zukunftsweisende Technologien, neue Geschäftsmodelle und Hautpflegeinnovationen voranzutreiben. Neben dem Investment in dermanostic investierte die OSCAR\&PAUL Beiersdorf Venture Capital Unit unter anderem im Mai 2021 in die personalisierte Hautpflegemarke Routinely. Die Marke setzt auf eine modulare Hautpflege-Routine, die basierend auf einem Online-Fragebogen und einer App die dynamische Beschaffenheit der Haut berücksichtigt. 